\title{
Tumor de Wilms: experiencia de 15 años en un Hospital de niños, Córdoba, Argentina
}

\author{
Wilms tumor: 15 years of experience at a children's hospital in Córdoba, \\ Argentina
}

\author{
Dra. Claudia Seminara ${ }^{a}$ Dra. M. Celia Planells ${ }^{a}$ Dr. Ramón E. Pogonza y Dra. Miriam Morales ${ }^{a}$ \\ Colaboradores: Dra. Celina Castro ${ }^{c}$, Dr. Ariel Sferco ${ }^{d}$, Dr. Arturo Sentagned, Dra. Melina Bertello $y$ \\ Dr. Roger Tisseraf
}

\begin{abstract}
RESUMEN
El objetivo de este trabajo fue describir la epidemiología, la presentación, el tratamiento y el seguimiento nefrológico de niños con tumor de Wilms. Se recopilaron datos de 46 pacientes. Se encontró baja edad de presentación ( $<40$ meses), con síntomas iniciales de dolor, masa abdominal y fiebre. La histología prevalente fue nefroblastoma tipo mixto. Todos los pacientes recibieron quimioterapia prequirúrgica seguida, en la mayoría de los casos, de nefrectomía unilateral. Los pacientes con alto riesgo histológico tuvieron un riesgo relativo de morir de 7,2 (IC $75 \%: 1,5-33,7)$ con respecto al resto y de recidiva de 2,5 (IC $75 \%: 1,0-6,4$ ). La sobrevida libre de enfermedad a 5 años fue del $70 \%$. El $80 \%$ mantuvo la función renal en estadio I al completar el tratamiento oncológico. El factor pronóstico más importante fue la histología. Estos pacientes requieren seguimiento nefrológico prolongado.

Palabras clave: tumor de Wilms, enfermedad renal crónica, nefrectomía.
\end{abstract}

http: / / dx.doi.org/10.5546/ aap.2019.263

Texto completo en inglés:

http:/ / dx.doi.org/10.5546/ aap.2019.eng.263

Cómo citar: Seminara C, Planells MC, Pogonza RE, Morales M. Tumor de Wilms: experiencia de 15 años en un Hospital de niños, Córdoba, Argentina. Arch Argent Pediatr 2019;117(4):263-266.

a. Servicio de Nefrología.

b. Servicio de Terapia Intensiva

Hospital de Niños de la Santísima Trinidad,

Córdoba, Argentina.

Colaboradores:

c. Servicio de Oncohematología.

d. Servicio de Cirugía.

Hospital de Niños de la Santísima Trinidad.

e. Residencia de Nefrología del Sanatorio Allende.

f. Residencia de Pediatría del Hospital de Niños de la Santísima Trinidad.

Correspondencia:

Dra. Claudia Seminara: claudiaseminara@hotmail.com

Financiamiento: Ninguno.

Conflicto de intereses: Ninguno que declarar.

Recibido: 20-4-2018

Aceptado: 9-1-2019

\section{INTRODUCCIÓN}

El tumor de Wilms es el tumor renal más frecuente en la edad pediátrica y representa el $6 \%$ del cáncer en los niños. Su incidencia es de 7,1 por millón de niños de entre 0 y 14 años. Thomas Rance lo describe por primera vez en 1814 y el cirujano alemán Max Wilms, en 1899, lo caracteriza como un tumor renal mixto con los tres componentes celulares (blastema, estroma y epitelio). ${ }^{1}$

Tiene presentación esporádica, aunque el 1-2 \% son familiares. Aproximadamente, el 5-7 \% son bilaterales y pueden ser sincrónicos (simultáneos) o metacrónicos (sucesivos)..$^{2-4}$

Está relacionado con mutaciones en la región 11 p13 (donde reside el gen WT1), en el brazo largo del cromosoma 16 y del cromosoma 1. Algunos de los tumores de Wilms se asocian a síndromes genéticos, como el de tumor de Wilms, aniridia, anomalías urogenitales y retraso mental (Wilms tumor, Aniridia, Genital abnormalities and Retardation; WAGR, por sus siglas en inglés), Denys-Drash y otros. ${ }^{4}$

La Sociedad Internacional de Oncología Pediátrica (SIOP) propone una clasificación de acuerdo con el riesgo y con el tipo histológico. En ella, se considera riesgo bajo (necrótico, blastomatosis), intermedio (epitelial, estromal, mixto y regresivo) y alto (blastemal, células claras y rabdoide). ${ }^{5}$

El tratamiento recomendado por la SIOP incluye quimioterapia prequirúrgica con el objetivo de reducir la masa tumoral y, así, simplificar la cirugía, disminuir el riesgo de ruptura tumoral y, por ende, la posibilidad de recaídas, y mejorar el pronóstico.,

La sobrevida de los pacientes con tumor de Wilms es mayor en comparación con otras neoplasias. ${ }^{3,5}$

Los criterios de estadificación del tumor de Wilms fueron definidos por el National Wilms Tumor Study-4 (NWTS-4) (Tabla 1). 
Estos pacientes precisan seguimiento nefrológico indefinido, debido a su condición de masa renal disminuida, por la aparición de toxicidades de acuerdo con el tratamiento recibido (quimioterapia y radioterapia) y por la posibilidad de aparición de segundos tumores. El riesgo de un segundo evento tumoral es de entre un $5 \%$ y un $7 \%$ a los 30 años. $^{7}$

El objetivo del presente trabajo fue describir la epidemiología, la forma de presentación, el tratamiento y el seguimiento nefrológico de los niños con tumor de Wilms atendidos en el Hospital de Niños de Córdoba desde enero de 2000 a diciembre de 2015 inclusive.

\section{POBLACIÓN Y MÉTODOS}

Estudio retrospectivo, observacional, basado en historias clínicas de pacientes con tumor de Wilms atendidos en el Hospital de Niños de La Santísima Trinidad desde 2000 a 2015 inclusive. Se incluyeron pacientes de 1 mes a 15 años de edad, de ambos sexos, con diagnóstico de tumor de Wilms uni- o bilateral, que recibieron tratamiento según la SIOP. ${ }^{6}$

Las variables fueron edad al momento del diagnóstico, sexo, síntomas en el debut, asociación con síndromes genéticos, compromiso uni- o bilateral, tipo histológico según la SIOP, estadificación según el NWTS-4 (Tabla 1), tratamiento quimioterápico, radioterapia, tratamiento quirúrgico (nefrectomía total, parcial o tumorectomía), tiempo entre el diagnóstico y la cirugía, evolución (recidiva y / o metástasis), hipertensión arterial (tensión arterial $>$ percentil 95 para sexo, edad y talla), ${ }^{8}$ proteinuria, ecografía renal (hipertrofia y alteraciones en la masa renal remanente/ recidivas), estadio de compromiso renal y mortalidad.

El estadio de compromiso renal se determinó según las guías Kidney Disease: Improving Global Outcomes (KDIGO) de 2012 sobre la base del filtrado glomerular estimado por la fórmula de Schwartz $(\mathrm{K} \times$ talla $/$ creatinina plasmática $=\mathrm{ml} /$ $\left.\mathrm{min} / 1,73 \mathrm{~m}^{2}\right)$. Se consideró filtrado normal mayor de $90 \mathrm{ml} / \mathrm{min} / 1,73 \mathrm{~m}^{2} .^{9}$ El control nefrológico y el de laboratorio fueron realizados una vez finalizada la quimioterapia. Los pacientes se evaluaron al año y a los 5 años del diagnóstico de tumor de Wilms.

Las variables categóricas se compararon usando las pruebas exactas de $\chi^{2}$ o Fisher, cuando correspondía, considerando como significativa una $\mathrm{P}<0,05$. Las variables continuas fueron descritas con el promedio, desvío estándar y cuartiles inferior y superior. Se utilizó el programa Epi Info 7.

El estudio fue aprobado por la Comisión Científica del Hospital de Niños.

\section{RESULTADOS}

Durante el período del estudio, se recopilaron datos de 46 pacientes con diagnóstico de tumor de Wilms. La edad promedio fue de 43 meses al momento del diagnóstico, con cuartil inferior de 17 y superior de 39 meses. Desvío estándar: 28,2 meses. Fueron femeninos 24 pacientes. Con respecto a la procedencia, 23 / 46 eran del interior de Córdoba; 12, de Córdoba capital, y 11, de otras provincias.

Los síntomas iniciales más frecuentes fueron tumoración abdominal en 26, dolor abdominal en 9 , fiebre en 8 y hematuria en 7 pacientes. De los

Tabla 1. Criterios de estatificación del National Wilms Tumor Study-4

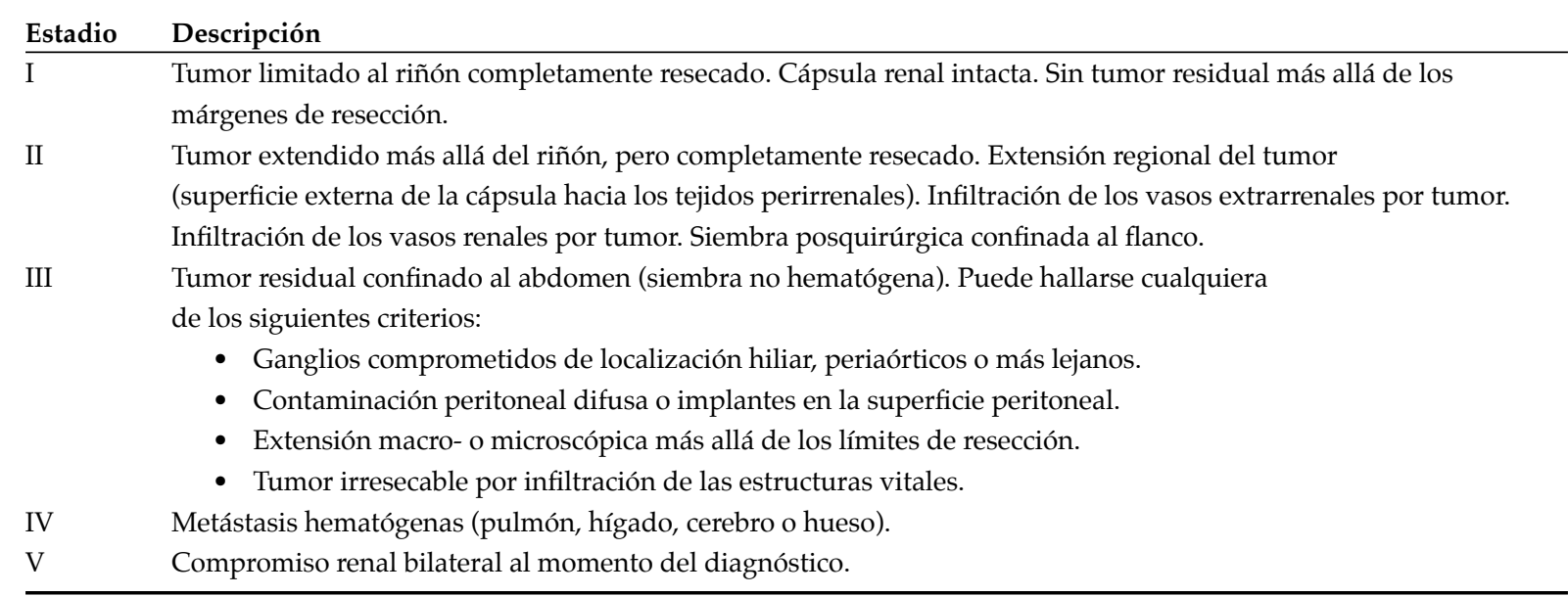


síndromes genéticos, solo se encontró el síndrome de WAGR en 3 pacientes, mientras que 1 paciente presentaba miocardiopatía idiopática previa al diagnóstico.

Con respecto a la localización renal, en 25 pacientes, fue derecha; en 19, izquierda y, en 2, bilateral. El diagnóstico fue clínico, por imágenes (ecografía y tomografía) y, en los casos de compromiso bilateral, con biopsia contralateral.

De acuerdo con el riesgo histológico, en 5 pacientes, era bajo; en 31, intermedio y, en 10, alto (Tabla 2). Con respecto a la estatificación del NWTS-4 al momento del diagnóstico, 23 pacientes correspondían al estadio I; 5, al II; 13, al III; 4, al IV, y 1, al V.

Todos los pacientes recibieron quimioterapia previa a la cirugía, que consistió en combinaciones de vincristina, actinomicina, doxorrubicina, etopósido, carboplatino e isofosfamida. Solo 11 pacientes recibieron radioterapia: 8 pacientes con riesgo histológico intermedio y 3 con alto riesgo.

El tratamiento quirúrgico consistió en la nefrectomía total en 39 pacientes, la nefrectomía parcial en 2, la nefrectomía unilateral más tumorectomía contralateral en 3, la tumorectomía en 1 y la heminefrectomía bilateral en 1 paciente.

Durante el seguimiento de 5 años de los 46 pacientes, 15 presentaron recidiva y/o metástasis de la enfermedad ( 2 pacientes de bajo riesgo, 8 de riesgo intermedio y 5 de alto riesgo). La sobrevida libre de enfermedad a los 5 años fue del $70 \%$. La supervivencia global a los 5 años fue del $87 \%$; la de los pacientes con bajo riesgo histológico, del $100 \%$, intermedio del $96 \%$ y alto del $33 \%$.

TABla 2. Distribución de los pacientes según el riesgo y el tipo histológico del tumor, sobre la base de la clasificación de la Sociedad Internacional de Oncología Pediátrica

\begin{tabular}{lclc}
\hline Riesgo & N & Histología & N \\
\hline Bajo riesgo & 6 & Necrótico & 3 \\
& & Blastomatosis & 3 \\
Riesgo intermedio & 27 & Epitelial & 5 \\
& & Estromal & 5 \\
& & Mixto & 13 \\
Alto riesgo & \multirow{2}{*}{13} & Regresivo & 4 \\
& & Blastemal & 9 \\
& & Células claras & 3 \\
& & Rabdoide & 1 \\
\hline
\end{tabular}

Del total de los pacientes atendidos en nuestra Institución, en 5 casos, no pudo hacerse el seguimiento renal por tratarse en otras instituciones y 6 pacientes fallecieron. En los 35 pacientes restantes, 5 presentaron proteinuria. En los controles ecográficos, se encontraron 19/35 pacientes con hipertrofia compensadora, 12 con riñón único con tamaño normal, 2 con tumor renal bilateral y 2 con malformación renal (riñón en herradura).

La función renal durante el seguimiento nefrológico (según la fórmula de Schwartz) fue la siguiente: 30 niños en estadio I, 4 en estadio II y 1 en estadio III.

Los pacientes con alto riesgo histológico presentaron un riesgo relativo (RR) de morir de 7,2 (intervalo de confianza-IC- $75 \%: 1,5-33,7$ ) con respecto a los de riesgo intermedio y bajo $(p=0,015)$; además, tuvieron un $\mathrm{RR}$ de recidiva de 2,5 (IC $75 \%: 1,0-6,4$ ).

\section{DISCUSIÓN}

Al momento del diagnóstico de tumor de Wilms, la mayoría de los pacientes tenían menos de 40 meses de edad. ${ }^{2}$ No hubo predominio de sexo y tampoco hubo diferencia significativa en cuanto a la lateralidad del riñón afectado.

Solo 3/46 pacientes presentaban síndrome genético, todos ellos WAGR, que no difirió de lo referido en trabajos previos. ${ }^{4}$

La presentación clínica más frecuente fue dolor abdominal y lumbar, seguida de masa abdominal, fiebre y hematuria, similar a otros estudios. ${ }^{10,11}$

El tipo histológico prevalente fue el nefroblastoma tipo mixto. En todos los pacientes, se realizó quimioterapia prequirúrgica seguida, en la mayoría de los pacientes, de nefrectomía total unilateral. El factor pronóstico más importante de sobrevida fue la histología según la SIOP; el de alto riesgo estuvo asociado a mayor mortalidad y recidivas.

La sobrevida global a 5 años del diagnóstico de nuestros pacientes fue del $87 \%$, ligeramente inferior a lo relatado por otras publicaciones, con más del $90 \% .^{12}$

Ningún paciente presentó insuficiencia renal terminal, aunque el tiempo de seguimiento de nuestros pacientes fue menor en comparación con otros centros, por lo que no se pudo hacer una comparación adecuada. ${ }^{13}$

En 5 de 35 pacientes, hubo filtrado glomerular menor de $90 \mathrm{ml} / \mathrm{min} / 1,73$ (estadios II-III de enfermedad renal crónica-ERC-). En solo 
8 pacientes, se encontró proteinuria, referida como un marcador temprano de ERC provocada por la nefrotoxicidad, la quimioterapia, la hiperfiltración y la radioterapia recibidas. ${ }^{14}$ No se realizó el seguimiento nefrológico, ya que los pacientes no fueron derivados a este Servicio.

Los continuos avances en el conocimiento de las bases genéticas y moleculares del tumor de Wilms permitirán establecer, en el futuro, terapias adaptadas al riesgo individualizado de cada paciente, así como identificar nuevas estrategias terapéuticas con un perfil de eficacia/toxicidad comparadas con la quimioterapia estándar. ${ }^{15}$

\section{CONCLUSIÓN}

La edad de presentación del tumor de Wilms en nuestros pacientes fue, principalmente, en menores de 40 meses, sin predominio de sexo. Los síntomas iniciales más frecuentes fueron dolor abdominal, masa abdominal, fiebre y hematuria. La sobrevida libre de enfermedad a los 5 años fue del $70 \%$.

Alrededor del $80 \%$ de nuestra población mantuvo la función renal en estadio I al completar el tratamiento oncológico. Es necesario un seguimiento nefrológico prolongado de estos pacientes, debido al riesgo de presentar, a mediano y a largo plazo, complicaciones secundarias a la enfermedad de base y al tratamiento recibido.

\section{REFERENCIAS}

1. Ries LAG, Melbert D, Krapcho M, Mariotto A, et al (eds.). SEER Cancer Statistics Review 1975-2004. Bethesda: National Cancer Institute; 2007. [Consulta: 11 de enero de 2019]. Disponible en: https://seer.cancer.gov/archive/ csr/1975_2004/.

2. Mambié Meléndez M, Guibelade del Castillo M, Nieto del RincónN, RodrigoJiménezD, etal. Tumor deWilms bilateral
Metacrónico. An Pediatric (Barc). 2002;56(3):247-50.

3. Mullen E, Weldon C, Kreidberg J. Pediatric Renal Tumors. In Avner E, Harmon W, Niaudet P, Yoshikawa N (eds.). Pediatric Nephrology.6thed.Heidelberg:Springer-Verlag; 2009:1431-55.

4. López L, Adragna M, Di Pinto D, Cacciavillano W, et al. Compromiso renal de los pacientes con Tumor de Wilms Bilateral. Med Infant. 2016;23:74-8.

5. Vujanić GM, Sandstedt B, Harms D, Kelsey A, et al. Revised International Society of Paediatric Oncology (SIOP) working classification of renal tumors of childhood. Med Pediatr Oncol. 2002;38(2):79-82.

6. Romao R, Lorenzo A. Renal function in patients with Wilms Tumor. Urol Oncol. 2016;34(1):33-41.

7. KasteSC, DomeJS, Babyn PS, Graf NM, etal. Wilms tumour: prognostic factors, staging, therapy and late effects. Pediatr Radiol. 2008;38(1):2-17.

8. National High Blood Pressure Education Program Working Group on High Blood Pressure in Children and Adolescent. The fourth report on the diagnosis, evaluation, and treatment of high blood pressure in children and adolescents. Pediatrics. 2004;114(2Suppl4th Report):555-76.

9. Stefanowicz J, Kosiak M, Romanowicz G, Owczuk R, et al. Glomerular filtration rate and prevalence of chronic kidney disease in Wilms' tumour survivors. Pediatr Nephrol. 2011;26(5):759-66.

10. Illadea L, Hernandez-Marques C, Cormenzana M, Lassaletta A, et al. Tumor de Wilms: revisión de nuestra experiencia en los últimos 15 años. An Pediatr (Barc). 2018:88(3):140-9.

11. Levitt G. Renal Tumours: Long-term outocome. Pediatr Nephrol. 2012;27(6): 911-6.

12. Isa ON, Reyes CM, Russo NM. Resultado del tratamiento del tumor de Wilms en población Pediátrica. Rev Chil Pediatr. 2013;84(6):628-34.

13. Kazama T, Nio M, Sasaki H, Fukuzawa T, Sato T. Estimated glomerular filtration rate after nephrectomy for Wilms tumor. Pediatr Int. 2018;60(10):962-5.

14. Skinner R. Late renal toxicity of treatment for childhood malignancy: risk factors, long-term outcomes, and surveillance. Pediatr Nephrol. 2018;33(2):215-25.

15. Dome JS, Graf N, Geller J, Fernandez C, et al. Advances in Wilms tumor treatment and biology: Progress Through internacional collaboration. J Clin Oncol. 2015;33(27):29993007. 